\title{
Manipulation of the Growth of Gold and Silver Nanomaterials on Glass by Seeding Approach
}

\author{
Kun-Hong Lee, ${ }^{\dagger}$ Kung-Ming Huang, ${ }^{\dagger}$ Wei-Lung Tseng, ${ }^{\ddagger}$ Tai-Chia Chiu, ${ }^{\dagger}$ \\ Yang-Wei Lin, ${ }^{\dagger}$ and Huan-Tsung Chang $*, \dagger, \S$ \\ Department of Chemistry, National Taiwan University, Taipei, Taiwan, Department of Chemistry, National \\ Sun Yat-Sen University, Kaohsiung, Taiwan, and Department of Natural Science Education, National \\ Taitung University, Taitung, Taiwan
}

Received June 29, 2006. In Final Form: October 13, 2006

\begin{abstract}
This paper describes the growth of gold and silver nanomaterials from their corresponding colloidal seeds on glass substrates. The glass substrates were treated with 3-mercaptopropyltrimethoxysilane (MPTMS) and then with gold or silver nanoparticle seeds (AuNPSs or AgNPSs) at different concentrations. After separately immersing the thusprepared MPTMS-AuNPS and MPTMS-AgNPS glass substrates in aqueous solutions containing cetyltrimethylammonium bromide, ascorbic acid, and gold or silver ions, we obtained $\mathrm{Au}$ and $\mathrm{Ag}$ nanomaterials of different sizes and shapes, which we characterized through scanning electron microscopy (SEM) and dark-field microscopy (DFM). At $0.001 \times$ concentrations AuNPSs and AgNPSs (i.e., 1000-fold-diluted solutions of the as-prepared samples), Au and $\mathrm{Ag}$ nanomaterials having 1.36 and $5.21 \mu \mathrm{m}$ lengths and aspect ratios of 11 and 24 were prepared in yields of $27 \%$ and 54\%, respectively. Scattering spectra of the thus-prepared single AuNRs and AgNRs revealed that the uniformity in the shape of the AuNRs was greater than that of the AgNRs. In addition, we demonstrate the preparation (using a laboratory-made synthesis system) and characterization (through DFM measurements) of Au nanomaterials, including AuNRs, from five AuNPS solutions of different concentrations on a single glass substrate.
\end{abstract}

\section{Introduction}

One-dimensional (1D) assemblies of nanoparticles, such as wires, rods, belts, and tubes, have attracted considerable attention because of their unique size- and shape-dependent optoelectronic and surface properties. ${ }^{1-4}$ Accordingly, it is widely expected that such nanomaterials will play a significant role in future electronic, optical, and mechanical devices. ${ }^{5-12}$ For example, semiconductor nanorods (NRs) and nanowires (NWs) have been tested for use as polarized light emission and lasing materials. ${ }^{13-15}$ Gold NRs (AuNRs) have extremely high absorptivity coefficients

* To whom correspondence should be addressed. Phone/fax: 011-8862-3366-1171. E-mail: changht@ntu.edu.tw.

National Taiwan University.

$\doteqdot$ National Sun Yat-Sen University.

$\S$ National Taitung University.

(1) Xia, Y.; Yang, P.; Sun, Y.; Wu, Y.; Mayers, B.; Gates, B.; Yin, Y.; Kim, F.; Yan, H. Adv. Mater. 2003, 15, 353.

(2) Huang, Y.; Chiang, C.-Y.; Lee, S. K.; Gao, Y.; Hu, E. L.; Yoreo, J. D.; Belcher, A. M. Nano Lett. 2005, 5, 1429.

(3) Hassenkam, T.; Moth-Poulsen, K.; Stuhr-Hansen, N.; Nørgaard, K.; Kabir,

M. S.; Bjørnholm, T. Nano Lett. 2004, 4, 19.

(4) Balakrishnan, K.; Datar, A.; Oitker, R.; Chen, H.; Zuo, J.; Zang, L. J. Am. Chem. Soc. 2005, 127, 10496.

(5) Bachtold, A.; Hadley, P.; Nakanishi, T.; Dekker, C. Science 2001, 294, 1317.

(6) Cui, Y.; Wei, Q.; Park, H.; Lieber, C. M. Science 2001, 293, 1289.

(7) Duan, X.; Niu, C.; Sahi, V.; Chen, J.; Parce, J. W.; Empedocles, S.; Goldman, J. L. Nature (London) 2003, 425, 274.

(8) Nicewarner-Peña, S. R.; Carado, A. J.; Shale, K. E.; Keating, C. D. J. Phys. Chem. B 2003, 107, 7360.

(9) Nicewarner-Peña, S. R.; Freeman, R. G.; Reiss, B. D.; He, L.; Peña, D. J.; Walton, I. D.; Cromer, R.; Keating, C. D.; Natan, M. J. Science 2001, 294 , 137.

(10) Keating, C. D.; Natan, M. J. Adv. Mater. 2003, 15, 451

(11) Burton, Z.; Bhushan, B. Nano Lett. 2005, 5, 1607.

(12) Jang, J. E.; Cha, S. N.; Choi, Y.; Amaratunga, G. A. J.; Kang, D. J.; Hasko, D. G.; Jung, J. E.; Kim, J. M. Appl. Phys. Lett. 2005, 87, 163114

(13) Johnson, J. C.; Yan, H.; Yang, P.; Saykally, R. J. J. Phys. Chem. B 2003, $107,8816$.

(14) Djurišić, A. B.; Kwok, W. M.; Leung, Y. H.; Phillips, D. L.; Chan, W. K. J. Phys. Chem. B 2005, 109, 19228. 15190

(15) Sirbuly, D. J.; Law, M.; Yan, H.; Yang, P. J. Phys. Chem. B 2005, 109 in the near-IR region, and therefore, they are suitable for use as sensing materials when conducting Raman scattering and absorbance measurements.

A number of approaches have been developed for preparing 1D nanomaterials, including electrochemical deposition in templates, electrochemical synthesis, photochemical synthesis, microwave heating, and seed-mediated growth. ${ }^{16-20}$ Seedmediated growth is a particularly efficient approach toward the synthesis of fairly monodispersed AuNRs and silver NRs (AgNRs) having high yields and different aspect ratios (AR). When AuNRs are prepared through seed-mediated growth, many parameters that control the particles' AR and yields must be controlled precisely, including the size and concentration of the metal colloidal seeds, the nature of the capping agents, the nature of the headgroup and the length of the chains of the selected surfactants, the reducing ability of the reducing agents, the $\mathrm{pH}$ and ionic strength of the solution, and the temperature. ${ }^{21-25} \mathrm{High}$ yields (ca. 97\%) of differently sized AuNRs with the average lengths ranging between 20 and $100 \mathrm{~nm}$ have been prepared after mixing gold colloidal seeds and a growth solution consisting of ascorbic acid, $\mathrm{AuCl}_{4}{ }^{-}$, and cetyltrimethylammonium bromide (CATB) ${ }^{26}$ Recently, we synthesized differently shaped and sized $\mathrm{Au}-\mathrm{Ag}$ core-shell NRs from 3.5-nm-diameter gold seeds by controlling the $\mathrm{pH}$ and glycine concentration under alkaline conditions ( $\mathrm{pH} 8.0-10.0){ }^{23,24}$

(16) Sander, M. S.; Gao, H. J. Am. Chem. Soc. 2005, 127, 12158.

(17) Yu, Y.-Y.; Chang, S.-S.; Lee, C.-L.; Wang, C. R. C. J. Phys. Chem. B 1997, 101, 6661.

(18) Kim, F.; Song, J. H.; Yang, P. J. Am. Chem. Soc. 2002, 124, 14316.

(19) Jiang, Y.; Zhu, Y.-J. J. Phys. Chem. B 2005, 109, 4361.

(20) Wei, Z.; Mieszawska, A. J.; Zamborini, F. P. Langmuir 2004, 20, 4322

(21) Gole, A.; Murphy, C. J. Chem. Mater. 2004, 16, 3633.

(22) Gao, J.; Bender, C. M.; Murphy, C. J. Langmuir 2003, 19, 9065.

(23) Huang, C.-C.; Yang, Z.; Chang, H.-T. Langmuir 2004, 20, 6089.

(24) Yang, Z.; Lin, Y.-W.; Tseng, W.-L.; Chang, H.-T. J. Mater. Chem. 2005 15,2450

(25) Gou, L.; Murphy, C. J. Chem. Mater. 2005, 17, 3668

(26) Sau, T. K.; Murphy, C. J. Langmuir 2004, 20, 6414. 
Similar to their aqueous suspensions, thin films made from AgNRs and AuNRs exhibit unique electronic and optical properties. For example, the metal-enhanced fluorescence (50fold) of human serum albumin labeled with indocyanine green has been demonstrated on AgNR-treated glass as has the promotion of electron-transfer reactions of $\left[\mathrm{Fe}(\mathrm{CN})_{6}\right]^{3-} /[\mathrm{Fe}-$ $\left.(\mathrm{CN})_{6}\right]^{4-} .27,28$ Layer-by-layer assembly ${ }^{29,30}$ and LangmuirBlodgett ${ }^{31,32}$ and electrochemical ${ }^{33,34}$ techniques are commonly used for the preparation of thin films of AgNRs and AuNRs. One example is the preparation of AuNRs thin films by dipping solid substrates modified with the gold nanoparticle seeds (AuNPSs) into growth solutions consisting of ascorbic acid, $\mathrm{AuCl}_{4}{ }^{-}$, and CATB. ${ }^{20,35,36}$ To ensure more uniform and complete surface coverage of AuNPSs on solid substrates, aminopropyltrimethoxysilane (APTMS) is often used to modify the surfaces of mica and $\mathrm{Si}$ substrates. The surface growth of AuNRs on an APTMStreated mica or $\mathrm{Si}$ substrate is terminated because of the accumulation of multilayers (or large aggregates having other forms) of CTAB around the surfaces of the growing particles; such accumulation blocks the access of gold precursor ions to the particle's metallic core. To understand why differently sized and shaped AuNRs are grown on 3-mercaptopropyltrimethoxysilane (MPTMS)-treated glass or silica wafer, the growth processes has been monitored directly through atomic force microscopy (AFM) and scanning electron microscopy (SEM) measurements. ${ }^{37,38}$ Preferential adsorption of CTAB on certain crystal faces best describes the growth of AuNRs with the initial seed structure playing an important role in determining whether a seed will grow into a NR or grows in only one or two directions; the size distribution of the seeds affects the dispersity of the surface-grown AuNRs. ${ }^{38}$ A study of the growth of AuNRs on poly-L-lysine-treated n-doped silicon wafers suggested that the seed particles must grow to a minimum diameter of $17 \mathrm{~nm}$ to initiate formation of AuNRs. ${ }^{37}$

Although there are several approaches for the syntheses of AuNRs and AgNRs on solid substrates, the direct, high-yield preparation of nanomaterials having a small size distribution remains a major challenge. In this study, we investigated the impact that the seed concentration has on the preparation of $\mathrm{Au}$ and Ag nanomaterials on glass substrates. In addition, we tested a multiplexed system for the synthesis of differently sized and shaped $\mathrm{Au}$ nanomaterials on a single MPTMS-treated glass substrate. Dark-field microscopy (DFM) and SEM measurements were conducted to characterize the thus-prepared nanomaterials. Our results suggest that the aspect ratios and size distributions of Au and Ag nanomaterials on MPTMS-treated glass substrates depend strongly on the concentrations of the AuNPSs and silver nanoparticle seeds (AgNPSs).

(27) Aslan, K.; Leonenko, Z.; Lakowicz, J. R.; Geddes, C. D. J. Phys. Chem. B 2005, 109, 3157 .

(28) Chang, G.; Zhang, J.; Oyama, M.; Hirao, K. J. Phys. Chem. B 2005, 109, 1204

(29) Gole, A.; Murphy, C. J. Chem. Mater. 2005, 17, 1325.

(30) Hu, X.; Cheng, W.; Wang, T.; Wang, Y.; Wang, E.; Dong, S. J. Phys. Chem. B 2005, 109, 19385

(31) Kim, F.; Kwan, S.; Akana, J.; Yang, P. J. Am. Chem. Soc. 2001, 123, 4360 .

(32) Tao, A.; Kim, F.; Hess, C.; Goldberger, J.; He, R.; Sun, Y.; Xia, Y.; Yang, P. Nano Lett. 2003, 3, 1229.

(33) Tian, M.; Wang, J.; Kurtz, J.; Mallouk, T. E.; Chan, M. H. W. Nano Lett. 2003, 3, 919 .

(34) Choi, J.; Sauer, G.; Nielsch, K.; Wehrspohn, R. B.; Gösele, U. Chem. Mater. 2003, 15, 776 .

(35) Taub, N.; Krichevski, O.; Markovich, G. J. Phys. Chem. B 2003, 107, 11579.

(36) Mieszawska, A. J.; Zamborini, F. P. Chem. Mater. 2005, 17, 3415.

(37) Liao, H.; Hafner, J. H. J. Phys. Chem. B 2004, 108, 19276.

(38) Wei, Z.; Zamborini, F. P. Langmuir 2004, 20, 11301.

\section{Experimental Section}

Chemicals. Sodium tetrachloroaurate(III) dehydrate, silver nitrate (99\%), and MPTMS were obtained from Sigma (St. Louis, MO). Sodium borohydride (powder, 99\%), sodium hydroxide, and trisodium citrate were purchased from Aldrich (Milwaukee, WI). 2-Propanol and CTAB (99\%) were obtained from Acros (New Jersey). L-Ascorbic acid was received from J. T. Baker (Philipsburg, NJ).

Functionalization of Glass Substrates. Glass microscope slides were immersed in piranha solution [30\% hydrogen peroxide and concentrated sulfuric acid (3:1)] overnight, and then the glass substrates were rinsed extensively with deionized water and dried under a stream of dry nitrogen prior to use. The cleaned slides were functionalized by dipping them for $30 \mathrm{~min}$ into solutions consisting of 2-propanol (10 mL), MPTMS (100 $\mu \mathrm{L})$, and deionized water $(5$ drops) at $60^{\circ} \mathrm{C}$. The MPTMS-coated glass slides were then rinsed with water and dried under a stream of nitrogen gas.

Growth of Au Nanomaterials on Glass Slides. Au nanomaterials were prepared on glass substrates according to a reported seedmediated growth method. ${ }^{20,38}$ The 3.5-nm-diameter AuNPSs were prepared by reduction of $\mathrm{HAuCl}_{4}$ using $\mathrm{NaBH}_{4} \cdot{ }^{39}$ The concentration of the thus-prepared AuNPSs was denoted as $1.0 \times$, which is about $1.1 \times 10^{14}$ particles $/ \mathrm{mL}$. The AuNPSs were then diluted with deionized water to achieve a final concentration range of $0.0001-$ $0.1 \times$. The MPTMS-treated glass substrates were placed directly (for $20 \mathrm{~min}$ ) into the aqueous solutions of the 3.5-nm-diameter AuNPSs. After removing the MPTMS-AuNPS-treated glass substrates from the solutions, the slides were rinsed with water and dried under a steam of dry nitrogen. Finally, they were placed in a $\mathrm{NR}$ growth solution at $28{ }^{\circ} \mathrm{C}$ for a certain length of time (from 10 min to $3 \mathrm{~h}$, as indicated). The NR growth solution consisted of 0.1 M CTAB $(9 \mathrm{~mL}), 0.01 \mathrm{M} \mathrm{NaAuCl}_{4}(450 \mu \mathrm{L}), 0.1 \mathrm{M}$ ascorbic acid $(50 \mu \mathrm{L})$, and deionized water $(500 \mu \mathrm{L})$.

Growth of Ag Nanomaterials on Glass Slides. Ag nanomaterials were prepared according to a procedure described previously. ${ }^{27}$ First, AgNPSs (diameter $4 \pm 2 \mathrm{~nm}$ ) were prepared by reducing silver nitrate with $\mathrm{NaBH}_{4}$ in the presence of sodium citrate. ${ }^{40}$ The concentration of the thus-prepared AgNPSs was denoted as $1.0 \times$ , which is about $7.7 \times 10^{13}$ particles $/ \mathrm{mL}$. Deionized water was used to dilute the original silver colloidal seeds to concentrations with the range $0.0001-0.1 \times$. The MPTMS-treated glass slides were directly immersed for $20 \mathrm{~min}$ in the AgNPS solutions ( $20 \mathrm{~mL}$ ). The MPTMSAgNPS-treated glass slides were then rinsed with water and dried under a steam of dry nitrogen. Finally, they were immersed for 5-60 min into a "growth solution" consisting of 0.1 M CTAB (20 $\mathrm{mL}), 0.1 \mathrm{M} \mathrm{AgNO}_{3}(50 \mu \mathrm{L}), 0.1 \mathrm{M}$ ascorbic acid $(1 \mathrm{~mL})$, and $1 \mathrm{M}$ $\mathrm{NaOH}(200 \mu \mathrm{L})$ at $28{ }^{\circ} \mathrm{C}$.

Multiplexed Synthesis System. Sylgard 184 silicone elastomer and a curing agent $[10: 1(\mathrm{w} / \mathrm{w})]$ were mixed and then degassed in a vacuum tank for $30 \mathrm{~min}$ to minimize formation of bubbles during curing. The mixture was poured into a template (stainless steel) containing five round holes $(0.9 \mathrm{~cm}$ in diameter), and then it was cured for $10 \mathrm{~min}$ in an oven at $70{ }^{\circ} \mathrm{C}$. The as-prepared poly(dimethylsiloxane) (PDMS) was then adhered to the MPTMS-treated glass. To ensure their contact, the assembly was heated for $10 \mathrm{~min}$ in an oven at $70{ }^{\circ} \mathrm{C}$. The five wells were then filled separately with AuNPS solutions of various concentrations $(0.0001-1.0 \times)$. After a reaction time of $20 \mathrm{~min}$, the wells were rinsed with deionized water, dried under nitrogen gas, and then filled with the growth solution of the Au nanomaterials. The mixture was reacted for $3 \mathrm{~h}$ at $28^{\circ} \mathrm{C}$. Finally, the PDMS was removed, and the glass was cleaned with water and dried under $\mathrm{N}_{2}$ gas.

Instrumentation. A double-beam UV-vis spectrophotometer (Cintra 10e) obtained from GBC (Victoria, Australia) was used to measure the absorbances of the $\mathrm{Au}$ and $\mathrm{Ag}$ nanomaterials both on glass substrates and in aqueous solutions. The sizes and shapes of the gold and silver nanomaterials were characterized using an SEM (ERA-8900FE) obtained from Elionix (Tokyo, Japan). The lab-

(39) Jana, N. R.; Gearheart, L.; Murphy, C. J. J. Phys. Chem. B 2001, 105, 4065.

(40) Jana, N. R.; Gearheart, L.; Murphy, C. J. Chem. Commun. 2001, 7, 617 
Scheme 1. Cartoon Depicting the Seed-Mediated Growth of NRs on MTPMS-treated Glass Substrates

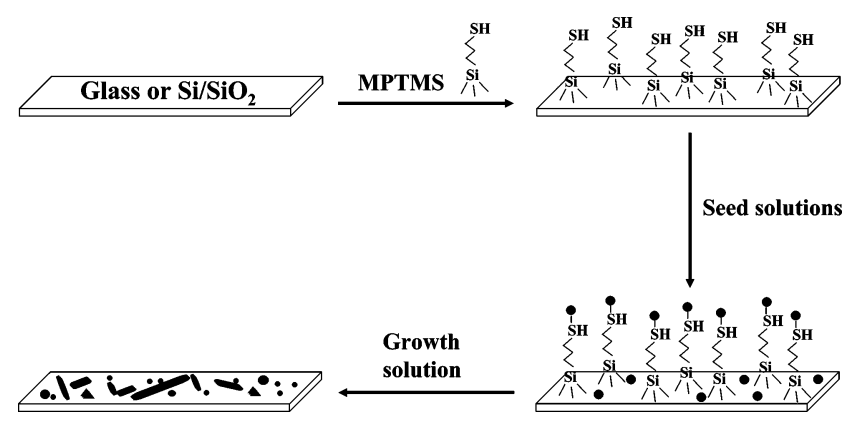

made DFM system consisted of an Olympus IX71 inverted microscope (Tokyo, Japan), an objective $(40 \times$; numerical apertures $=0.75)$, a high-resolution $(4086 \times 2048$ pixels $)$ digital camera (DP70, Olympus, Tokyo, Japan), a 100-W halogen lamp, and a condenser (IX-ULWCD, Olympus, Japan). Image J and Sigmascan Pro 5.0 software were used to analyze the images. With an aperture of $500 \times 500 \mu \mathrm{m}^{2}$, the scattering spectra of single NRs were recorded using a spectrometer (Ocean optics 2100, Ocean Optics Inc., Duiven, The Netherlands).

\section{Results and Discussion}

Effect of Seed Concentration on the Growth of Au and Ag Nanomaterials. Scheme 1 summarizes the synthesis of $\mathrm{Au}$ nanomaterials and Ag nanomaterials on MPTMS-treated glass substrates through seed-mediated growth. The growth of NRs directly on surfaces is different from that performed in aqueous solution for a number of reasons. For example, the substrate blocks the diffusion of the $\mathrm{AuCl}_{2}-\mathrm{CTAB}$ complexes on one face of the seeds/NRs; in addition, no seed particle Ostwald ripening or aggregation occurs. ${ }^{38}$ Despite these differences, the parameters that affect the shapes, sizes, and yields of NRs grown on surfaces and in aqueous solution are quite similar. ${ }^{20,37}$ In previous reports, ${ }^{22,39,40}$ we suggested that aqueous solutions consisting of CTAB $(0.1 \mathrm{M})$, ascorbic acid $(0.5 \mathrm{mM})$, and $\mathrm{HAuCl}_{4}$ $(0.25 \mathrm{mM})$ or $\mathrm{AgNO}_{3}(0.25 \mathrm{mM})$ provide high yields and small size dispersities for preparations of $\mathrm{Au}$ nanomaterials and $\mathrm{Ag}$ nanomaterials. Thus, we selected such solutions for a study exploring the effects that the AuNPS and AgNPS concentrations have on the shapes and sizes of the Au nanomaterials and Ag nanomaterials, respectively, which were prepared on MPTMStreated glass substrates.

Initially, we tested the impact that the concentration $(0.0001-$ $1.0 \times$; see the Experimental Section for definition) of AuNPSs has on the preparation of Au nanomaterials on MPTMS-treated glass substrates. After dipping MPTMS-treated glass substrates in different AuNPS solutions at ambient temperature and pressure for $20 \mathrm{~min}$, we obtained MPTMS-AuNPS-treated glass substrates. The surface density of Au seeds was estimated from the corresponding SEM images, which were 207.6, 21.0, 7.7, 2.9, $1.1 \times 10^{7}$ particles $/ \mathrm{cm}^{2}$ when using $1 \times, 0.1 \times, 0.01 \times, 0.001 \times$, and $0.0001 \times$ seed solutions, respectively. Upon immersing them in a growth solution for $10 \mathrm{~min}$, the color of the MPTMSAuNPS-treated glass substrates began to turn from colorless to pink, indicating formation of Au nanomaterials on the surfaces. Because the colors (absorbance) of the glass substrates did not undergo any further change after ca. $3 \mathrm{~h}$, in this study we conducted all of the reactions for $3 \mathrm{~h}$. The SEM images exhibited in Figure $1 \mathrm{a}-\mathrm{c}$ display the formation of differently shaped Au nanomaterials on the surfaces, including Au nanomaterials (0.77-1.72 $\mu \mathrm{m}$ in length), spherical (0.13-0.19 $\mu \mathrm{m}$ in diameter), triangular (0.21 $-0.59 \mu \mathrm{m}$ in base length), hexagonal $(0.30-0.47 \mu \mathrm{m}$ in base length), and others. Figure 1d displays the UV-vis spectra
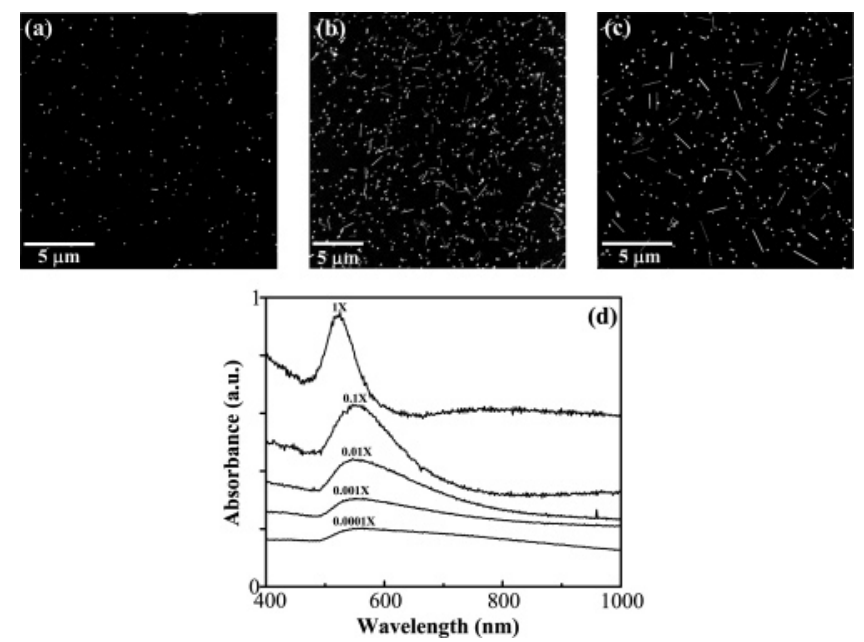

Figure 1. SEM images indicating the impact that the AuNPS concentration has on the preparation of $\mathrm{Au}$ nanomaterials on MPTMS-AuNPS-treated glass substrates. (a, b, and c) AuNPS concentrations of $1.0 \times, 0.01 \times$, and $0.0001 \times$, respectively. (d) UVvis absorption spectra of the corresponding gold nanomaterials. The MPTMS-AuNPS-treated glass substrates were immersed for $3 \mathrm{~h}$ in growth solutions containing $0.1 \mathrm{M} \mathrm{CTAB}, 0.5 \mathrm{mM} \mathrm{NaAuCl}_{4}$, and $0.5 \mathrm{mM}$ ascorbic acid at $28{ }^{\circ} \mathrm{C}$.

of the Au nanomaterials formed on the MPTMS-AuNPS-treated glass substrates. The bands at ca. $520 \mathrm{~nm}$ are characteristic of the surface plasmon resonance (SPR) absorption (extinction) of spherical Au nanomaterials and the transverse SPR absorption of Au nanomaterials. ${ }^{20}$ Because Au nanomaterials of different shapes and sizes were prepared, these SPR bands appear broad. Upon decreasing the AuNPS concentration from $1.0 \times$ to $0.0001 \times$ , the transverse SPR absorption bands shifted to longer wavelength because of the formation of longer Au nanomaterials (cf. Figure $1 \mathrm{a}-\mathrm{c})$. The longitudinal SPR absorption bands of Au nanomaterials undergo red shifts when the aspect ratio increases. The bands corresponding to the longitudinal SPR absorptions of $\mathrm{Au}$ nanomaterials having aspect ratios higher than 6 were not visible and detected by our spectrometer because they occurred in the near-IR region (at wavelengths above $1000 \mathrm{~nm}$ ). ${ }^{41}$ Table 1 indicates that the lengths and widths of the Au nanomaterials both increased upon decreasing the concentration of the AuNPSs. When the concentrations of AuNPSs decreased from $1.0 \times$ to $0.001 \times$, the aspect ratios decreased (from 12 to 18 to 8 to 13 , respectively) and yields increased (from $5 \pm 3 \%$ to $25 \pm 2 \%$, respectively). By controlling the AuNPS concentrations, the Au nanomaterials were prepared with higher yields $(23-27 \%)$ when compared to that by conducting a previously reported method $(4-14 \%) .{ }^{20}$ To understand the impact that the concentration of AuNPSs plays on formation of the Au nanomaterials, the mean surface coverage and mean distances among AuNPS at different concentrations of AuNPSs are listed in Table 2. Because of the shorter distances among AuNPS and more spherical AuNPSs in certain surface area at higher AuNPS concentrations, several AuNPS tend to grow to bigger spherical AuNPs after they grew to certain sizes. On the other hand, at low AuNPS concentrations there are fewer AuNPSs on the surface, and thus, they have less chance to grow to greater spherical AuNPs. In other words, each AuNPS grew to a longer $\mathrm{Au}$ nanomaterial after deposition of $\mathrm{Au}$ atoms. ${ }^{26,39,42,43}$ We note that the $\{111\}$ facet of the $\mathrm{Au}$ nanomaterials is more accessible to gold atoms than is the $\{110\}$ facet, upon which densely packed CTAB assemblies form.

(41) Nikoobakht, B.; El-Sayed, M. A. Chem. Mater. 2003, 15, 1957.

(42) Jana, N. R.; Gearheart, L.; Murphy, C. J. Adv. Mater. 2001, 13, 1389.

(43) Murphy, C. J.; Jana, N. R. Adv. Mater. 2002, 14, 80. 
Table 1. Impact of AuNPS Concentration on the Preparation of Au Nanomaterials on MPTMS-treated Glass Substrates ${ }^{a}$

\begin{tabular}{lccccc}
\hline & \multicolumn{4}{c}{ AuNPS concentration $(\times)$} \\
\cline { 2 - 5 } & 1 & 0.1 & 0.01 & 0.001 & 0.0001 \\
\hline length $(\mu \mathrm{m})$ & $0.97 \pm 0.20$ & $1.13 \pm 0.26$ & $1.24 \pm 0.24$ & $1.36 \pm 0.22$ & $1.52 \pm 0.20$ \\
width $(\mathrm{nm})$ & $66 \pm 17$ & $95 \pm 26$ & $99 \pm 30$ & $130 \pm 35$ & $155 \pm 31$ \\
AR & $14.7 \pm 2.9$ & $11.9 \pm 2.9$ & $12.5 \pm 3.1$ & $10.5 \pm 2.1$ & $9.8 \pm 1.4$ \\
yield $(\%)^{b}$ & $5.1 \pm 3.2$ & $17.7 \pm 0.5$ & $21.1 \pm 2.1$ & $25.2 \pm 2.2$ & $20.7 \pm 2.6$
\end{tabular}

${ }^{a}$ Reaction conditions are the same as those described in Figure 1. ${ }^{b}$ Five batches.

Table 2. Impact of AuNPS and AgNPS Concentrations on the Mean Distance and Surface Coverage Percentages of the Corresponding Seeds

\begin{tabular}{rlrrrrr}
\hline & & \multicolumn{4}{c}{ seed concentration $(\times)$} \\
\cline { 3 - 6 } & & \multicolumn{1}{c}{1} & \multicolumn{1}{c}{0.1} & \multicolumn{1}{c}{0.01} & 0.001 & \multicolumn{1}{c}{0.0001} \\
\hline \multirow{2}{*}{$\mathrm{Au}$} & distance $(\mu \mathrm{m})^{a}$ & $0.3 \pm 0.1$ & $0.7 \pm 0.3$ & $1.3 \pm 0.4$ & $2.9 \pm 1.1$ & $4.5 \pm 1.4$ \\
& coverage $(\%)^{b}$ & $75.4 \pm 1.4$ & $51.0 \pm 1.5$ & $27.7 \pm 1.7$ & $9.8 \pm 2.5$ & $4.5 \pm 2.1$ \\
$\mathrm{Ag}$ & distance $(\mu \mathrm{m})$ & $1.2 \pm 0.2$ & $2.4 \pm 0.9$ & $3.6 \pm 1.4$ & $5.2 \pm 2.2$ & $6.4 \pm 2.8$ \\
& coverage $(\%)$ & $67.7 \pm 1.8$ & $41.0 \pm 1.2$ & $21.6 \pm 1.5$ & $4.8 \pm 1.8$ & $2.7 \pm 1.7$
\end{tabular}

${ }^{a}$ Mean distances are estimated from AuNPSs and AgNPSs $(n=500) .{ }^{b}$ The surface coverage percentages were calculated from five different batches
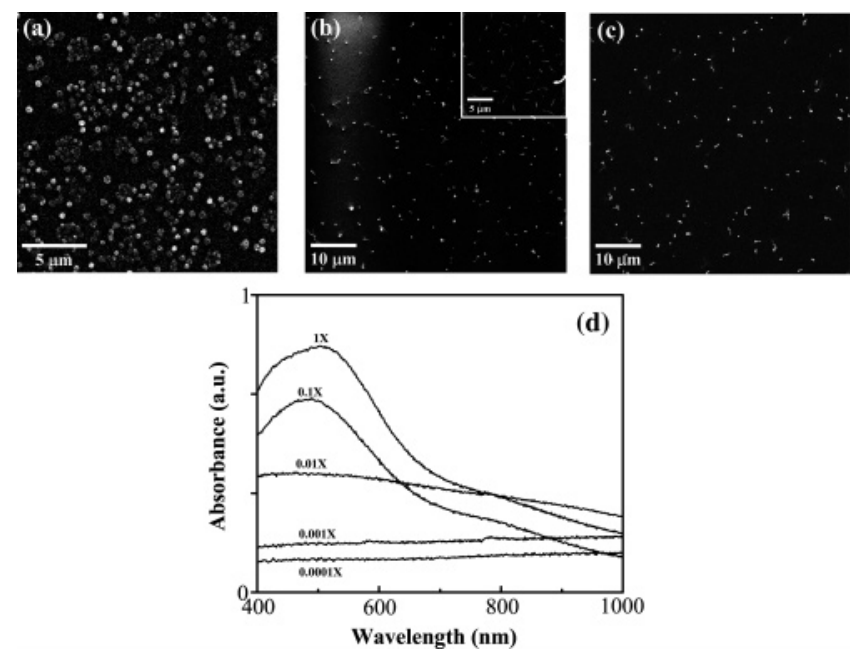

Figure 2. SEM images indicating the impact that the AgNPS concentration has on the preparation of $\mathrm{Ag}$ nanomaterials on MPTMS-AgNPS-treated glass substrates. (a, b, and c) AgNPS concentrations of $1.0 \times, 0.001 \times$, and $0.0001 \times$, respectively. The inset shows a 10-fold-amplified SEM image of the Ag nanomaterials. (d) UV-vis absorption spectra of the corresponding silver nanomaterials. The MPTMS-AgNPS-treated glass substrates were immersed for $1 \mathrm{~h}$ in growth solutions containing $0.1 \mathrm{M} \mathrm{CTAB}, 0.25$ $\mathrm{mM} \mathrm{AgNO} 3,5.0 \mathrm{mM}$ ascorbic acid, and $0.01 \mathrm{M} \mathrm{NaOH}$ at $28^{\circ} \mathrm{C}$.

The SEM images depicted in Figure $2 \mathrm{a}-\mathrm{c}$ clearly indicate formation of silver nanomaterials of different shapes and sizes on the MPTMS-AgNPS-treated glass substrates, including spherical nanomaterials, tadpole-like nanomaterials, and nanowires. Figure $2 \mathrm{~d}$ displays the UV-vis absorption spectra of the corresponding silver nanomaterials. The surface densities of AgNPSs were estimated from the corresponding SEM images, which were $11.2,5.7,4.0,3.4$, and $2.2 \times 10^{7}$ particles $/ \mathrm{cm}^{2}$ when using $1 \times, 0.1 \times, 0.01 \times, 0.001 \times$, and $0.0001 \times$ seed solutions, respectively. At high AgNPS concentrations $(>0.01 \times)$, the SPR bands appeared at ca. $500 \mathrm{~nm}$, which were attributed to spherical Ag nanomaterials. The diameters of spherical Ag nanomaterials that were prepared at $1.0 \times, 0.1 \times$, and $0.01 \times \mathrm{AgNPS}$ concentrations were 91, 180, and $260 \mathrm{~nm}$, respectively (Figure 2a). In addition, we did not observe any other shapes of Ag nanomaterials under those conditions. At low concentrations $(<0.01 \times)$ we did not observe the SPR bands at ca. $500 \mathrm{~nm}$ but formation of $\mathrm{Ag}$ nanomaterials with large aspect ratios as exhibited in Figure $2 \mathrm{~b}$

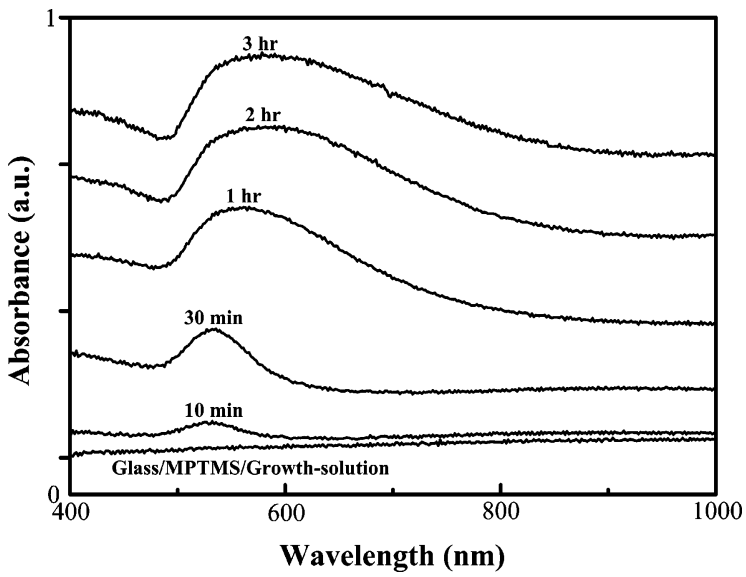

Figure 3. UV-vis absorption spectra of Au nanomaterials prepared on MPTMS - AuNPS-treated glass substrates for different times (10$180 \mathrm{~min})$. The AuNPS concentration was $0.001 \times$. Other conditions were the same as those described in Figure 1.

and c. The SEM image in Figure 2b indicates that, in addition to spherical Ag nanomaterials, Ag nanomaterials having lengths greater than $3.90 \mu \mathrm{m}$ and widths of ca. $0.11 \mu \mathrm{m}$ (AR values $>$ 22 ) were prepared with a yield of $54 \%$ at $0.001 \times$ AgNPS, which is greater when compared to that by conducting a previously reported method (10\%). ${ }^{27}$ Importantly, the SEM image exhibited in the inset in Figure $2 \mathrm{~b}$ displays that the thus-prepared $\mathrm{Ag}$ nanomaterials have a narrow size distribution ( $<30 \%$ in length). The longitudinal SPR absorption for the Ag nanomaterials having such high aspect ratios occurs in the near-IR region. Upon decreasing the AgNPS concentration further, the amounts and lengths of the Ag nanomaterials both decreased. For example, at $0.0001 \times$ AgNPSs (Figure 2c), the yield of Ag nanomaterials having lengths greater than $3.2 \mu \mathrm{m}$ and widths of ca. $0.26 \mu \mathrm{m}$ $(\mathrm{AR}>12)$ was $46 \%$. We believe that formation of such long $\mathrm{Ag}$ nanomaterials is likely to be the result of the coalescence of several Ag nanomaterials (see below). ${ }^{44}$

Growth Kinetics of Au and Ag Nanomaterials. No Au nanomaterials or Ag nanomaterials formed in the absence of AuNPSs or AgNPSs on the glass substrates, suggesting that the seeds act as nucleation sites or catalysts for gold or silver deposition. Figure 3 presents the UV-vis absorption spectra of

(44) Wei, G.; Zhou, H.; Liu, Z.; Song, Y.; Wang, L.; Sun, L.; Li, Z. J. Phys Chem. B 2005, 109, 8738 . 
Table 3. Impact of Time on the Preparation of Au Nanomaterials on MPTMS-Treated Glass Substrates ${ }^{a}$

\begin{tabular}{lcccc}
\hline & \multicolumn{3}{c}{ Reaction time $(\mathrm{min})$} \\
\cline { 2 - 5 } & 30 & 60 & $1.17 \pm 0.24$ & 180 \\
\hline length $(\mu \mathrm{m})$ & $0.74 \pm 0.17$ & $1.11 \pm 0.20$ & $130 \pm 21$ & $1.39 \pm 0.35$ \\
width $(\mathrm{nm})$ & $118 \pm 29$ & $114 \pm 21$ & $9.2 \pm 2.2$ & $126 \pm 22$ \\
AR & $6.7 \pm 2.5$ & $10.2 \pm 3.0$ & $12.5 \pm 3.9$ &
\end{tabular}

${ }^{a}$ The AuNPS concentration was $0.001 \times$. Other conditions were the same as those described in Figure 1 .
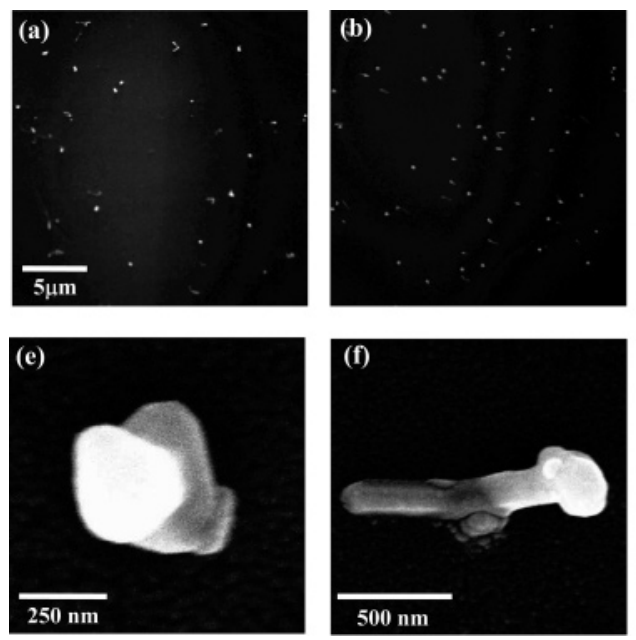
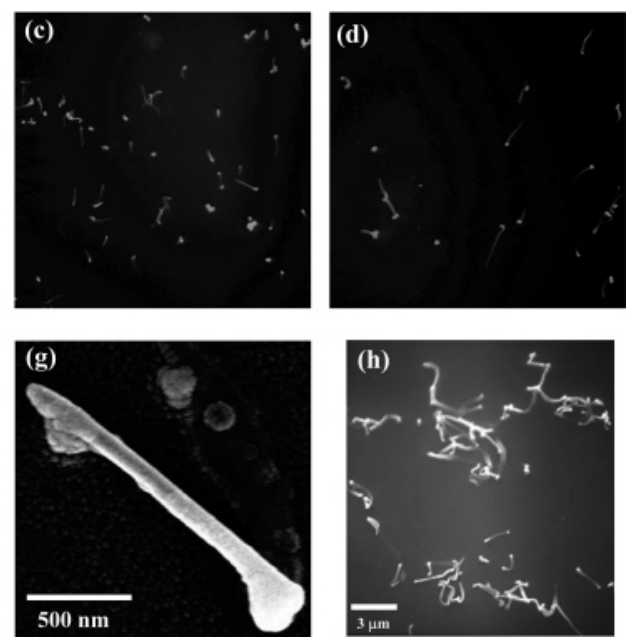

Figure 4. SEM images of Ag nanomaterials prepared on MPTMS-AgNPS-treated glass substrates. (a, b, c, and d) Reaction times of 5, 10, 20, and $40 \mathrm{~min}$, respectively. (e, f, and g) Amplified (60 000-fold) SEM images of Ag nanomaterials after reactions for 20, 40, and 60 min, respectively. (h) SEM image of different lengths of Ag nanomaterials after equilibrium (60 min). The AgNPS concentration was $0.001 \times$.

Table 4. Impact of Preparation Time on the Formation of Ag Nanomaterials on MPTMS-Treated Glass Substrates ${ }^{a}$

\begin{tabular}{lccccc}
\hline & \multicolumn{5}{c}{ Reaction time $(\mathrm{min})$} \\
\cline { 2 - 5 } & 5 & 10 & 20 & 40 & 60 \\
\hline length $(\mu \mathrm{m})$ & $1.34 \pm 0.16$ & $2.26 \pm 0.32$ & $3.72 \pm 0.63$ & $4.95 \pm 0.76$ & $5.21 \pm 1.31$ \\
width $(\mu \mathrm{m})$ & $0.17 \pm 0.07$ & $0.20 \pm 0.07$ & $0.20 \pm 0.08$ & $0.21 \pm 0.09$ & $0.22 \pm 0.11$ \\
AR & $7.5 \pm 2.1$ & $11.1 \pm 1.7$ & $18.5 \pm 4.0$ & $23.2 \pm 4.0$ & $23.7 \pm 4.9$
\end{tabular}

${ }^{a}$ The AgNPS concentration was $0.001 \times$. Other conditions were the same as those described in Figure 2 .

the gold nanomaterials prepared in growth solution after different periods of time (10-180 min) from AuNPS $(0.001 \times)$. During the first $30 \mathrm{~min}$, the intensities of the transverse SPR bands increased and their maximum wavelength (ca. $532 \mathrm{~nm}$ ) changed slightly, indicating that the number of gold atoms deposited on the AuNPS to form the $\mathrm{Au}$ nanomaterials (including $\mathrm{Au}$ nanomaterials) increased upon increasing reaction time. Table 3 indicates that the change in the length of the Au nanomaterials was not proportional to the reaction time. The average growth rates for the lengths of the $\mathrm{Au}$ nanomaterials over the reaction times in the ranges $0-30,30-60,60-120$, and $120-180 \mathrm{~nm}$ were ca. $25,12,1$, and $4 \mathrm{~nm} / \mathrm{min}$, respectively. The widths of the Au nanomaterials increased rapidly in the preparation course of 0-30 min, slowly 30-60 min, quickly again between 60 and $120 \mathrm{~min}$, and finally kept constant. We note that the yields (ca. $25 \%$ ) of the Au nanomaterials remained almost constant after $30 \mathrm{~min}$. These results suggest that the Au nanomaterials grew in two directions during the first $30 \mathrm{~min}$, and then (30-60 min) they gradually grew in one direction (long axis) to increase their lengths. Finally (60-120 min) they grew in other direction (short axis) to increase their widths. These results are in good agreement with those reported previously in the literature. ${ }^{20} \mathrm{CTAB}$ is believed to adsorb as a bilayer that selectively stabilizes and reduces growth along the $\mathrm{Au}\{100\}$ and $\mathrm{Au}\{110\}$ facets of the Au nanomaterials to promote AuNR growth at the ends. ${ }^{38,45}$ With higher concentrations of $\mathrm{AuCl}_{2}{ }^{-}-\mathrm{CTAB}$ complexes on one side of the AuNPSs/

(45) Nikoobakht, B.; El-Sayed, M. A. Langmuir 2001, 17, 6368.
Au nanomaterials, growth along the short axis was slower than that along the long axis. After increasing the length and decreasing the concentration of $\mathrm{AuCl}_{4}{ }^{-}$in the growth solution, accumulation of $\mathrm{AuCl}_{2}{ }^{-}-\mathrm{CTAB}$ complexes along the short axis abated and growth to wider width became more favorable.

Unlike the different shapes of the Au nanomaterials grown at $0.001 \times$ AuNPS, Ag nanomaterials were dominant at $0.001 \times$ AgNPSs. Thus, growth of the Ag nanomaterials appeared to be quite different from that of the Au nanomaterials. The SEM image depicted in Figure 4a clearly displays that there were many spheres and some tadpole-like Ag nanomaterials after incubation for $5 \mathrm{~min}$. Upon increasing the reaction time from 5 to $40 \mathrm{~min}$, the number of spheres and tadpole-like Ag nanomaterials decreased, but more Ag nanomaterials were formed (Figure $4 b-d$ ). Table 4 indicates that the widths of the $\mathrm{Ag}$ nanomaterials remained almost constant $(220 \mathrm{~nm})$ after $10 \mathrm{~min}$, but the lengths increased from 1.34 to $5.21 \mu \mathrm{m}$ between reaction times of 5 and $60 \mathrm{~min}$ with the corresponding aspect ratios increasing from 8 to 24 . It is important to note that each $\mathrm{Ag}$ nanomaterial had a big headgroup, indicating that the AgNPSs had to grow into a spherical Ag nanomaterial of a certain size (minimum size: $334 \pm 75 \mathrm{~nm}$ ) before growth of the $\mathrm{Ag}$ nanomaterials occurred in one direction. The amplified SEM images depicted in Figure $4 \mathrm{e}-\mathrm{g}$ indicate that the Ag nanomaterials grew from spherical to hook-shaped and then to NRs during the course of the reaction between 20 and 60 min. Although the straight Ag nanomaterials remained prominent, the amounts of 


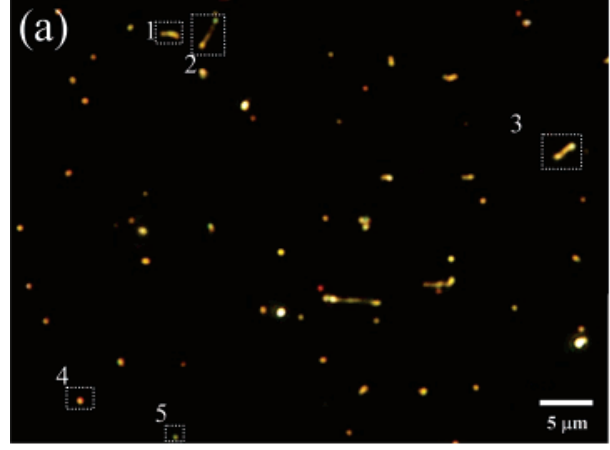

(c)

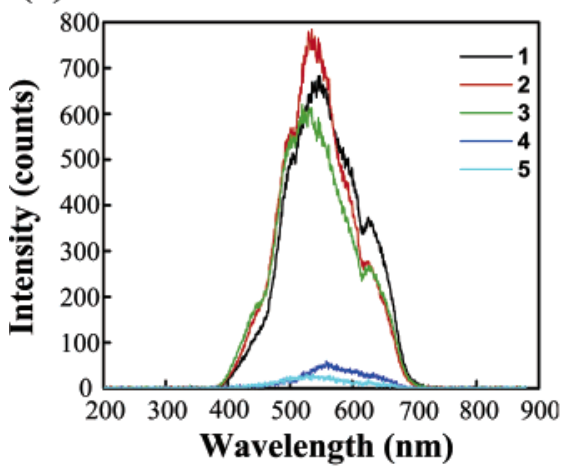

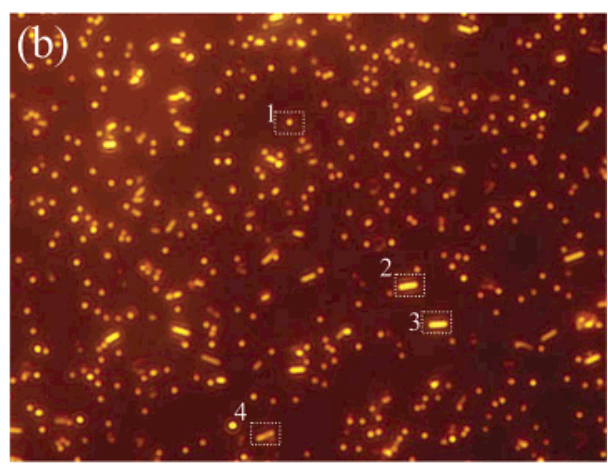

(d)

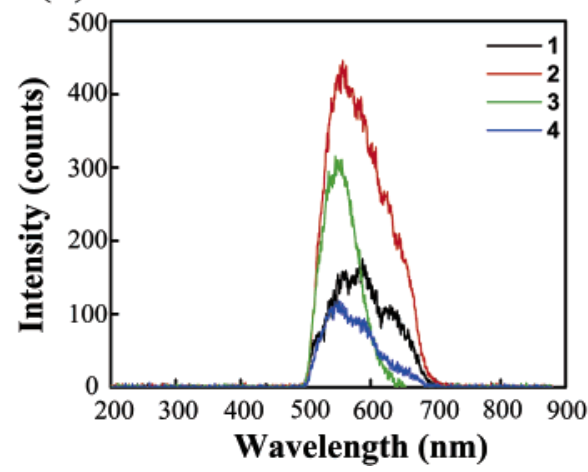

Figure 5. DFM images of (a) Ag and (b) Au nanomaterials prepared on MPTMS-AgNPS-treated glass substrates. (c and d) Scattering spectra of single nanomaterials. The concentrations of AgNPS and AuNPS were both $0.001 \times$. Other conditions were the same as those described in Figures 2 and 1, respectively.

curved and branched particles increased at low AgNPS concentrations. At low concentrations the distances between the seeds on the MPTMS-treated glass substrate were relatively long (see Table 2). Because there was more unoccupied (free) space on the surface for the seeds, their distribution was also relatively random when compared to that obtained at high seed concentrations. As a result, growth of the AgNPSs to differently shaped and sized Ag nanomaterials increased. The effect that the concentration of AgNPSs has on formation of these differently shaped and sized Ag nanomaterials is similar to that observed previously for the concentration dependence of ascorbic acid on formation of $\mathrm{Ag}$ nanomaterials from AgNPSs on indium tin oxide substrates. ${ }^{28}$ The surface coverage of AgNPS at the concentration of $0.001 \times$ was much lower than that at $0.01 \times$, and thus, the mean distances among interparticles increased (Table 2). As a result of deposition of Ag atoms, AgNPSs grew to certain sizes and then grew in one direction. Once adjacent Ag nanomaterials grew to certain sizes, they emerged and formed long $\mathrm{Ag}$ nanomaterials $(3.9-6.5 \mu \mathrm{m})$. Our reasoning is supported by formation of different lengths of Ag nanomaterials as shown in the SEM image (Figure 4h). With further decreasing the AgNPS concentration to $0.0001 \times$, the yield of long Ag nanomaterials decreases. Since the mean distance between any two adjacent AgNPSs is about $6.4 \mu \mathrm{m}$, there is a lesser chance for several AgNPs to form long Ag nanomaterials. On the contrary, when the concentrations of AgNPSs were greater than $0.01 \times$, the AgNPSs are much closer and have greater chance to form larger spherical AgNPs as they grew to certain sizes.

DFM Images and UV-vis Spectra for Single Ag and Au Nanomaterials. The DFM images of nanomaterials and their corresponding SPR absorption spectra are highly dependent on the particles' sizes and shapes, material compositions, and local environment. The DFM images depicted in Figure 5a and $b$ clearly display formation of differently shaped and sized Ag and Au nanomaterials when using $0.001 \times$ AgNPS and AuNPS, respectively. In contrast with the single color appearing in the image of the single Au nanomaterials, multiple colors appear for each Ag nanomaterial, reflecting their nonuniform structures. One application of DFM measurement is to allow singlenanoparticle-based SPR measurements to be performed with the advantage of great sensitivity to small changes in the nature of the environment. ${ }^{46,47}$ Figure $5 \mathrm{c}$ displays the scattering spectra of five single-particle Ag nanomaterials that are either spherical or rod-like, namely, marked 1-5 in Figure 5a. The scattering spectra clearly indicate that the Ag nanomaterials (particles 1-3) and particles 4 and 5 exhibited SPR bands at ca. $500 \mathrm{~nm}$, but their longitudinal SPR bands were not observed because of their high aspect ratios; these results are similar to those obtained in liquid solutions. The spectra also indicate that single Ag nanomaterials have greater absorptivity coefficients than do the single spherical Ag nanomaterials; again, these results are similar to those observed in liquid solution. The bands for particles $1-3$ are assigned to their transverse SPR absorptions because the scattering of longitudinal SPR absorption bands could not be observed for Ag nanomaterials having aspect ratios above 8 . The scattering spectra for the single Au nanomaterials and spherical Au nanomaterials exhibited in Figure 5d indicate that the SPR bands for these nanomaterials were both centered at $560 \mathrm{~nm}$, a similar value to those bands observed for spherical Au nanomaterials (180-200 $\mathrm{nm}$ in diameter) in liquid solutions. ${ }^{48}$ The results are in good agreement with our estimation of the width of Au nanomaterials $(179-215 \mathrm{~nm})$ and the diameter $(184-213 \mathrm{~nm})$ of spherical $\mathrm{Au}$ nanomaterials from scattering images. We assign the bands for particles 2-4 to the transverse SPR absorptions of the Au

(46) Raschke, G.; Kowarik, S.; Franzl, T.; Sönnichsen, C.; Klar, T. A.; Feldmann, J.; Nichtl, A.; Kürzinger, K. Nano Lett. 2003, 3, 935.

(47) McFarland, A. D.; Van Duyne, R. P. Nano Lett. 2003, 3, 1057.

(48) Yguerabide, J.; Yguerabide, E. E. Anal. Biochem. 1998, 262, 137 

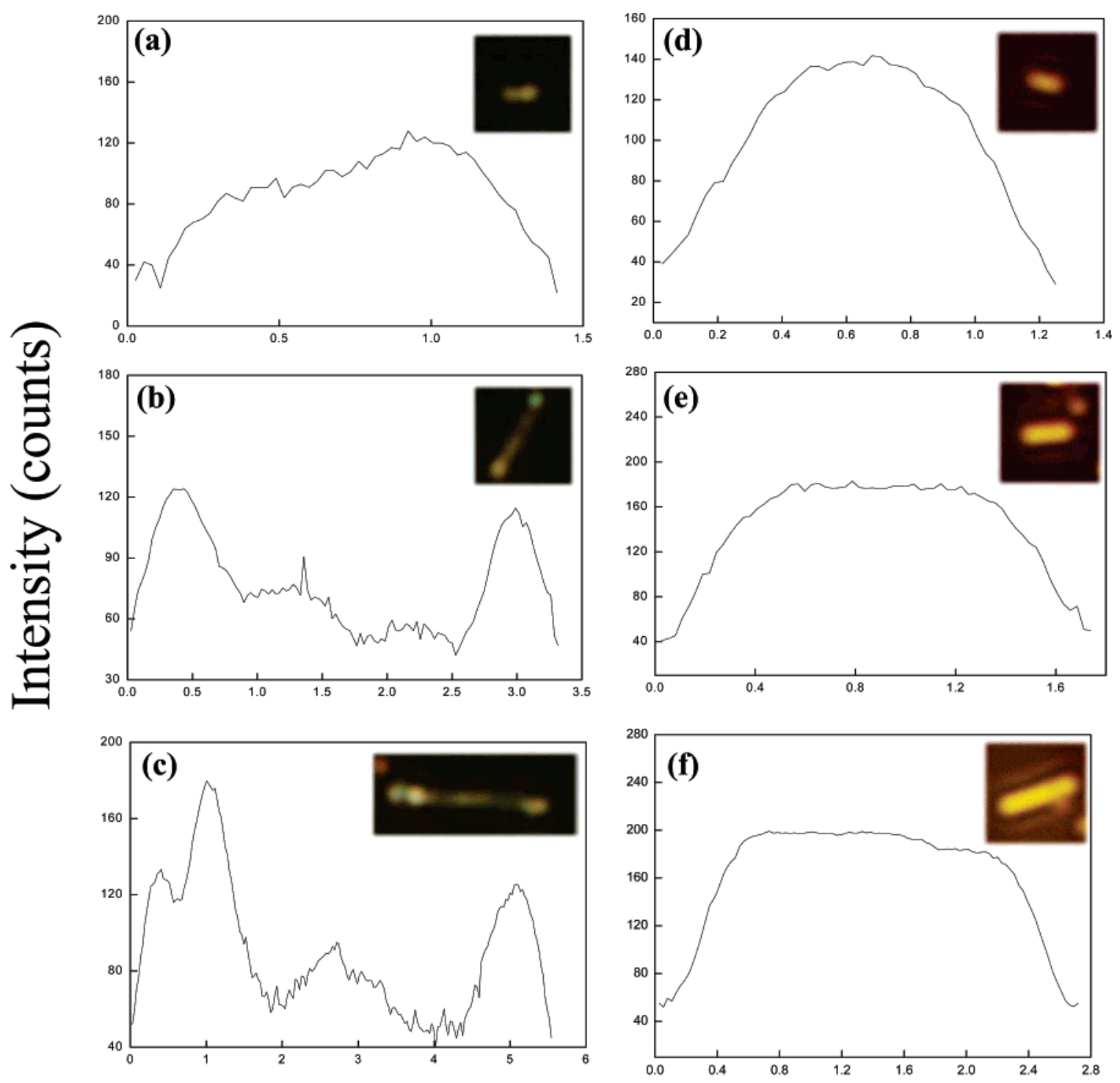

\section{Length $(\mu \mathrm{m})$}

Figure 6. Scattering intensities recorded at different positions of the $(\mathrm{a}-\mathrm{c})$ single $\mathrm{Ag}$ nanomaterials and $(\mathrm{d}-\mathrm{f})$ Au nanomaterials. The intensity is plotted against the position of the focusing points in each single nanomaterial.

nanomaterials. We note that the spectra exhibited in Figure 5c and $\mathrm{d}$ both appear quite rough because of the poor resolution of the CCD.

The differences between the single Ag and Au nanomaterials are further exhibited by the scattering intensities of the single NRs in different regions (Figure 6). The single Ag nanomaterials display different scattering intensities along their long axis, while those for $\mathrm{Au}$ nanomaterials are quite constant. The multicolor images for the single Ag nanomaterials are due to the different scattering properties (intensities and wavelengths) of single $\mathrm{Ag}$ nanomaterials of various widths; i.e., the many colors indicate the nonuniformity of the Ag nanomaterials with respect to their shape. The nonuniformity of these Ag nanomaterials supports our suggestion that formation of $\mathrm{Ag}$ nanomaterials is likely to occur through coalescence of several Ag nanomaterials. The aspect ratios of the NRs observed in the DFM images are smaller than these values measured through SEM imaging, mainly because the optically imaged width of a NR is diffraction limited. The diffraction-limited value is equal to ca. $\lambda / 2 \mathrm{NA}$, where $\lambda$ is the wavelength of the light and NA is the value of the numerical aperture of the object. When using an objective with a value of NA of 0.75 and light having a wavelength of $400-600 \mathrm{~nm}$, the diffraction limits fall in the range $267-400 \mathrm{~nm}$, which is ca. 2-3 times larger than the physical width of the $\mathrm{Ag}$ nanomaterials and $\mathrm{Au}$ nanomaterials. ${ }^{49}$ The widths of the corresponding $\mathrm{Ag}$ nanomaterials and Au nanomaterials measured through SEM imaging were $220 \pm 110$ and $130 \pm 35 \mathrm{~nm}$, respectively.

Multiplexed Synthesis of Au Nanomaterials. Figure 7 displays the results we obtained when using a laboratory-made synthetic system for the simultaneous preparation of $\mathrm{Au}$ nanomaterials on single MPTMS-treated glass substrates exposed to different concentrations of AuNPSs. As expected, we observed different colors - similar to those mentioned above-from the five AuNPS solutions of different concentrations. The DFM images clearly indicate that the lengths and yields of the Au nanomaterials increased when the AuNPS concentration decreased from $1.0 \times$ to $0.0001 \times$. This result suggests that use of

(49) Mock, J. J.; Oldenburg, S. J.; Smith, D. R.; Schultz, D. A.; Schultz, S. Nano Lett. 2002, 2, 465. 

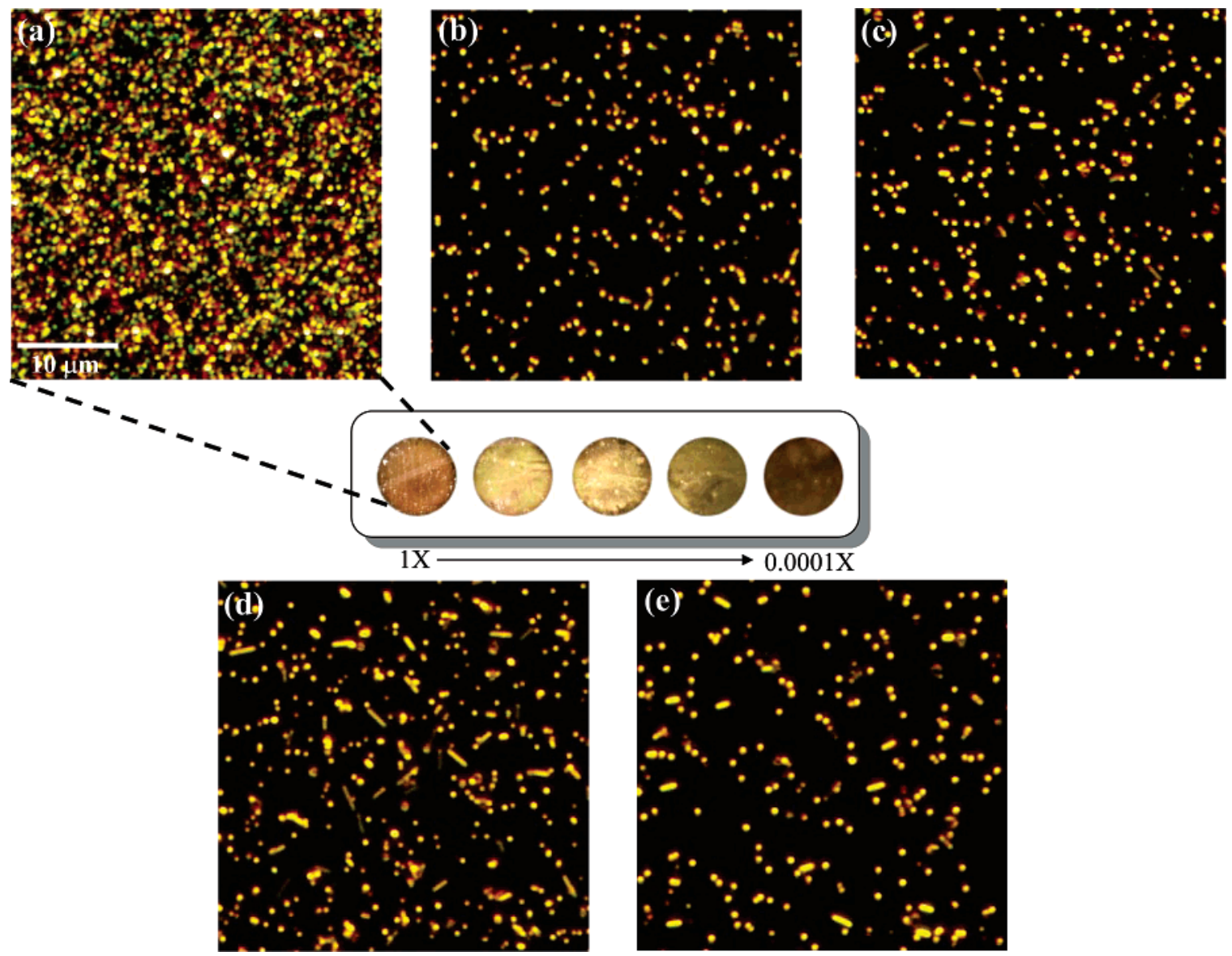

Figure 7. Multiplexed synthesis of Au nanomaterials on an MPTMS-AuNPS-treated glass substrate. The AuNPS concentrations were within the range $1-0.0001 \times$, with 10-fold dilution occurring in the wells from left to right. $(\mathrm{a}-\mathrm{e})$ Amplified (400-fold) DFM images recorded from the individual wells.

our approach, in conjunction with a DFM system, allows potential for high-throughput synthesis and characterization of nanomaterials on solid substrates. By controlling the concentration and nature of both the particle seeds and the growth solution, we should be able to prepare nanopatterns of various nanomaterials that might hold great potential for use in electronics systems. ${ }^{50,51}$

\section{Conclusions}

In this study we investigated the effects that the AuNPS and AgNPS concentrations have on the preparation of their corresponding nanomaterials on glass substrates. By carefully controlling the AuNPS and AgNPS concentrations, we prepared $\mathrm{Au}$ and $\mathrm{Ag}$ nanomaterials having different sizes and shapes with up to $27 \%$ and $54 \%$ yields for $\mathrm{Au}$ nanomaterials and $\mathrm{Ag}$ nanomaterials, respectively. Using a laboratory-made synthetic system, in conjunction with DFM, we were able to prepare and analyze a range of different Au nanomaterials on a single glass substrate from solutions of AuNPSs having five different

(50) Briseno, A. L.; Aizenberg, J.; Han, Y.-J.; Penkala, R. A.; Moon, H.; Lovinger, A. J.; Kloc, C.; Bao, Z. J. Am. Chem. Soc. 2005, 127, 12164.

(51) Cui, Y.; Björk, M. T.; Liddle, J. A.; Sönnichsen, C.; Boussert, B.; Alivisatos, A. P. Nano Lett. 2004, 4, 1093. concentrations. Using this system, characterization of nanomaterials does not depend on AFM and SEM instruments that are difficult to access for most laboratories. Our results clearly demonstrate that the nature of the growth of $\mathrm{Au}$ and $\mathrm{Ag}$ nanomaterials on glass substrates is different. We obtained Ag nanomaterials having a narrow size distribution, higher yields, and larger sizes (5.2 $\mu \mathrm{m}$ in length). The AgNPSs grew into spherical Ag nanomaterials having diameters greater than a threshold value $(334 \pm 75 \mathrm{~nm})$ prior to growing into long $\mathrm{Ag}$ nanomaterials; in addition, the AuNPSs grew in two directions to form NRs of certain lengths before first increasing their width and then their length in one direction. The thus-prepared $\mathrm{Au}$ nanomaterials were much smoother (uniform) than the $\mathrm{Ag}$ nanomaterials.

Acknowledgment. This study was supported by the National Science Council of Taiwan under contract NSC 95-2113-M002-026-MY3. We thank the NTU Information and Electronic Technology Center for providing the SEM measurements. T.-C.C. is grateful to the National Science Council for his postdoctoral fellowship (NSC 95-2811-M-002-051).

LA061880J 